\title{
VALUE ADDED TAX - VAT A CENTENARIAN [1920-2020] IN THIS COVID-19
}

\author{
Dr. Hariharan Narayanan ${ }^{* 1} \mathbb{\text { iD }}$ \\ *1 Founder and Director, Shri Academy, Pune, Maharashtra, India \\ ${ }^{*}$ Visiting Faculty, Department of Commerce \& Research Center, Savitribai Phule Pune University, \\ Pune, Maharashtra, India
}

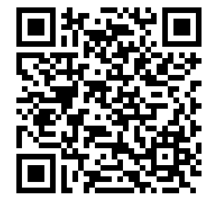

DOI: https://doi.org/10.29121/granthaalayah.v8.i9.2020.1323

Article Type: Research Article

Article Citation: Dr. Hariharan Narayanan. (2020). VALUE ADDED

TAX - VAT A CENTENARIAN [1920-

2020] IN THIS COVID-19.

International Journal of Research GRANTHAALAYAH, 8(9), 29-42.

https://doi.org/10.29121/granthaa

layah.v8.i9.2020.1323

Received Date: 25 August 2020

Accepted Date: 24 September 2020

Keywords:

VAT

Value Added Tax

GST

Consumption Tax

Standard Rate

European Union

\begin{abstract}
Value Added Tax is an indirect tax which is also termed as Goods and Service Tax. Both these terms are used in the same meaning in different parts of the world. VAT is followed almost in many countries and during this pandemic situation many countries have relaxed VAT for the benefit of the public. This study mainly focuses on the frameworks followed by various nations regarding VAT. It concentrates on the origin, growth and development of VAT, various tax model frameworks followed in nearly 147 different countries all around and the various measures adopted by countries towards VAT during this era of COVID-19 pandemic.
\end{abstract}

\section{INTRODUCTION}

VAT refers to Value Added Tax. In some countries it is known as GST, Goods and Services Tax. It is a type of indirect tax which is assessed incrementally. This tax is charged on the added value of a product or service at each stage of production, distribution and selling to consumers. VAT, GST and Consumption $\operatorname{Tax}^{1}$ are at times used interchangeably. It is a type of tax which is calculated at multiple stages or at multiple points, meaning calculated at each stage or point. Usually countries which adopt VAT scheme require all its businesses to get registered for VAT purposes based on the turnover of businesses. VAT registration becomes compulsory if the turnover crosses a specified limit, and this limit of turnover differs from country to country. VAT registered businesses can be natural persons or legal entities. VAT is usually collected by the end retailer and it is usually a flat tax. In many countries VAT generates a key revenue to the government. Even though many countries favour VAT, the concept has its own flaw

${ }^{1}$ It is an indirect tax which is charged on the consumption spending of goods and services. Stamp Tax, Tax on Tea and Whiskey Taxes will speak more about the History of Consumption Tax. More reference can be seen in this link https://en.wikipedia.org/wiki/Consumption_tax

(C) 2020 The Author(s). This is an open access article distributed under the terms of the Creative Commons Attribution License, which permits unrestricted use, distribution, and reproduction in any medium, provided the original author and source are credited. 
as quoted by the sub-national governments. This study will bring out a picture about the origin, concept, growth and evolution of VAT globally, the rates and systems followed by various countries and the measures adopted by different countries towards VAT during this COVID-19 era. The researcher felt that this is the right time to study VAT along with the measures adopted by various countries across the globe.

\section{OBJECTIVES}

- To study the origin, growth and evolution of VAT

- To study the Standard VAT rates followed globally

- To study the various Tax systems followed by different countries (Global Tax System)

- To study various measures adopted globally towards VAT / GST during COVID-19

\section{MATERIALS AND METHODS}

This study is carried with Secondary Data from various authentic sources. The study is historical, descriptive and analytical in nature. Since the data are based on secondary source any biased information on the secondary data may reflect on the study which should be taken as a limitation.

\section{DISCUSSION AND RESULTS}

\subsection{ORIGIN, EVOLUTION AND GROWTH}

(Ebrill., et al., 2001) German businessman Wilhelm Von Siemens was the intellect who came up with the idea of a VAT in 1920s. During 1920s other suggestions of invoice-credit method by Adams also peeped. Value-added tax or VAT first introduced less than 50 years ago, is now a vital factor of tax schemes around the world. The fast and clearly alluring rise of the VAT is possibly the most extensive tax development of the latter twentieth century. VAT was invented by Maurice Laure (French Economist), Joint Director of the French Tax Authority in 1954. From 10 th April 1954 it was introduced for large business and then extended to all business sectors in France. In French it was known as TVA. The following gives a picture about the rates prevailed till 2001 as given by IMF and extracted from the mentioned source

Table 1: VAT Rates till 2001

\begin{tabular}{|c|c|c|c|}
\hline Date of Introduction & Number of Countries & Single Rate & Multiple Rates \\
\hline Before 1990 & 48 & 12 & 36 \\
\hline $1990-1994$ & 46 & 31 & 15 \\
\hline 1995 - April 2001 & 31 & 25 & 05 \\
\hline Total & 125 & 68 & 56 \\
\hline
\end{tabular}

Source: IMF - The Modern VAT

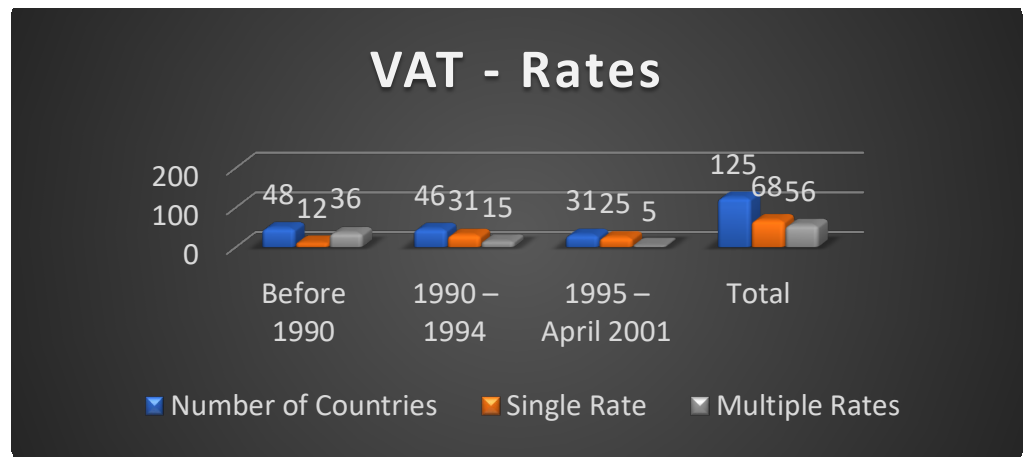

Figure 1

Source: Table 01 
Dr. Hariharan Narayanan

\subsection{GLOBAL TAX MODEL FOLLOWED}

Table 2: Sketch of Global Tax System with Covid Measure Rates

\begin{tabular}{|c|c|c|c|c|c|c|}
\hline $\begin{array}{l}\text { S. } \\
\text { No. }\end{array}$ & Country & $\begin{array}{c}\text { Tax } \\
\text { Model }\end{array}$ & E-A-L-Name ${ }^{2}$ & Year $^{3}$ & $\mathrm{SR}^{4}$ & Registration $^{5}$ \\
\hline 1 & Afghanistan & VAT & -- & 2014 & $10 \%$ & Afn150 million \\
\hline 2 & Albania & VAT & $\begin{array}{l}\text { TVSH - Tatimi mbi } \\
\text { Vleren e Shtuar }\end{array}$ & April 1995 & $20 \%$ & ALL2 million \\
\hline 3 & Algeria & VAT & $\begin{array}{l}\text { TVA - Taxe sur la } \\
\text { Valeur Ajoutee }\end{array}$ & April 1992 & $19 \%$ & $\begin{array}{l}\text { Apply for Lump } \\
\text { sum tax regime. } \\
\text { DZD30 million } \\
\text { (not in the scope } \\
\text { of VAT) }\end{array}$ \\
\hline 4 & Andorra & VAT & -- & $\begin{array}{c}\text { January } \\
2013 \\
\end{array}$ & $4.5 \%$ & $\begin{array}{l}\text { Compulsory for } \\
\text { all taxpayers }\end{array}$ \\
\hline 5 & Angola & VAT & $\begin{array}{l}\text { IVA - Imposto sobre } \\
\text { o Valor Acrescentado }\end{array}$ & July 2019 & $14 \%$ & None \\
\hline 6 & Argentina & $\begin{array}{l}\text { VAT } \\
\text { IIBB }\end{array}$ & $\begin{array}{l}\text { IVA - Impuesto al } \\
\text { Valor Agregado }\end{array}$ & $\begin{array}{l}\text { VAT }-1975 \\
\text { IIBB }-1977\end{array}$ & $21 \%$ & $\begin{array}{l}\text { ARS134 million } \\
\text { for goods and } \\
\text { ARS89 million for } \\
\text { services }\end{array}$ \\
\hline 7 & Armenia & VAT & -- & $\begin{array}{c}\text { January } \\
2018 \\
\end{array}$ & $20 \%$ & AMD15 million \\
\hline 8 & Aruba & $\begin{array}{l}\text { RT } \\
\text { HT }\end{array}$ & $\begin{array}{l}\text { Revenue Tax } \\
\text { Health Tax }\end{array}$ & $\begin{array}{c}\text { January } \\
2007 \text { and } \\
\text { July } 2018 \\
\text { December } \\
2014 \\
\end{array}$ & $3 \%$ for both & $\begin{array}{c}\text { For all taxpayers } \\
\text { who do business } \\
\text { in Aruba }\end{array}$ \\
\hline 9 & Australia & GST & -- & July 2000 & $10 \%$ & $\begin{array}{c}\text { AUD75,000 } \\
(150,000 \text { for } \\
\text { nonprofit bodies) }\end{array}$ \\
\hline 10 & Austria $^{6}$ & VAT & -- & $\begin{array}{c}\text { January } \\
1973\end{array}$ & $19 \%$ and $20 \%$ & $\begin{array}{c}\text { EUR30,000 } \\
\text { (Established in } \\
\text { Austria) } \\
\text { Nil (Established } \\
\text { outside Austria }\end{array}$ \\
\hline 11 & Azerbaijan & VAT & DV - Deyer Vergisi & $\begin{array}{c}\text { January } \\
1992 \\
\end{array}$ & $18 \%$ & AZN200,000 \\
\hline 12 & Bahamas & VAT & -- & $\begin{array}{c}\text { January } \\
2015 \\
\end{array}$ & $7.5 \%$ and $12 \%$ & BSD100,000 \\
\hline 13 & $\begin{array}{l}\text { Kingdom of } \\
\text { Bahrain }\end{array}$ & VAT & -- & $\begin{array}{c}\text { January } \\
2019 \\
\end{array}$ & $5 \%$ & BHD37,500 \\
\hline 14 & Bangladesh & VAT & -- & $\begin{array}{c}\text { July } 1991 \\
\text { and } \\
\text { Amended on } \\
\text { July } 2017\end{array}$ & $15 \%$ & $\begin{array}{l}\text { Taka } 03 \text { million } \\
\text { And } \\
\text { (Proposed is } \\
\text { BDT } 30 \text { million) }\end{array}$ \\
\hline
\end{tabular}

2 Expansion, Alternate or Local Name

${ }^{3}$ Year of Introduction

${ }^{4}$ Standard Rate in Percentage

${ }^{5}$ Registration Thresholds (In Annual Turnover)

${ }^{6}$ Has reduced VAT rate due to COVID-19 to 50\% of the standard rate applicable from 01 July to 31 December 2020 International Journal of Research -GRANTHAALAYAH 
Value Added Tax - Vat A Centenarian [1920-2020] in This Covid-19

\begin{tabular}{|c|c|c|c|c|c|c|}
\hline 15 & Barbados & VAT & -- & $\begin{array}{c}\text { January } \\
1997\end{array}$ & $17.5 \%$ & BBD200,000 \\
\hline 16 & Belarus & VAT & $\begin{array}{c}\text { NDS - Nalog na } \\
\text { Dobavlennuyu } \\
\text { Stoimost }\end{array}$ & $\begin{array}{c}\text { December } \\
1991\end{array}$ & $20 \%$ & None \\
\hline 17 & Belgium & VAT & $\begin{array}{c}\text { BTW - Belasting over } \\
\text { de Toegevoegde } \\
\text { Waarde } \\
\text { TVA - Taxe sur la } \\
\text { Valeur Ajoutee } \\
\end{array}$ & $\begin{array}{c}\text { January } \\
1972\end{array}$ & $21 \%$ & $\begin{array}{l}\text { EUR35,000 } \\
\text { (Distance } \\
\text { Selling0 }\end{array}$ \\
\hline 18 & Bolivia & VAT & $\begin{array}{l}\text { IVA - Impuesto al } \\
\text { Valor Agregado }\end{array}$ & July 1986 & $14 \%$ & None \\
\hline 19 & $\begin{array}{l}\text { Bonaire, Sint } \\
\text { Eustatius and } \\
\text { Saba (BES } \\
\text { Islands) }\end{array}$ & GET & $\begin{array}{c}\text { General Expenditure } \\
\text { Tax }\end{array}$ & $\begin{array}{c}\text { January } \\
2011\end{array}$ & $\begin{array}{l}\text { Between 6\% } \\
\text { and } 8 \%\end{array}$ & None \\
\hline 20 & Botswana & VAT & & July 2002 & $12 \%$ & BWP01 million \\
\hline 21 & Brazil & $\begin{array}{c}\text { ICMS } \\
\text { IPI } \\
\text { ISS } \\
\text { PIS- } \\
\text { PASEP } \\
\text { /COFINS }\end{array}$ & $\begin{array}{l}\text { ICMS - State VAT } \\
\text { IPI - Federal VAT } \\
\text { ISS - Municipal } \\
\text { service tax } \\
\text { PIS-PASEP/COFINS - } \\
\text { Federal gross receipt } \\
\text { contributions }\end{array}$ & $\begin{array}{c}\text { ICMS - } 1989 \\
\text { IPI - } 1964 \\
\text { ISS - } 1968 \\
\text { PIS-PASEP - } \\
1970 \\
\text { COFINS - } \\
1991\end{array}$ & $\begin{array}{l}\text { ICMS- } 0 \% \text { to } \\
35 \% \\
\text { IPI- } 0 \% \text { to } \\
300 \% \\
\text { ISS- } 0 \% \text { to } 5 \% \\
\text { PIS-PASEP } \\
0.65 \% \text { to } \\
1.65 \% \\
\text { COFINS } \\
3 \% \text { to } 7.6 \% \\
\end{array}$ & None \\
\hline 22 & Bulgaria $^{7}$ & VAT & $\begin{array}{l}\text { DDS - Danak varhu } \\
\text { Dobavenata Stoinost }\end{array}$ & April 1994 & $20 \%$ & BGN50,000 \\
\hline 23 & Cameroon & VAT & -- & $\begin{array}{c}\text { January } \\
1999\end{array}$ & $19.5 \%$ & XAF50 million \\
\hline 24 & Canada & $\begin{array}{l}\text { GST } \\
\text { HST }\end{array}$ & $\begin{array}{c}\text { Goods and Services } \\
\text { Tax } \\
\text { Harmonized Services } \\
\text { Tax }\end{array}$ & $\begin{array}{c}\text { GST January } \\
1991 \\
\text { HST April } \\
1997\end{array}$ & $\begin{array}{c}\text { GST }-5 \% \\
\text { HST }-13 \% \text { to } \\
15 \%\end{array}$ & CAD30,000 \\
\hline 25 & Chile & VAT & $\begin{array}{l}\text { IVA - Impuesto al } \\
\text { Valor Agregado }\end{array}$ & $\begin{array}{c}\text { December } \\
1974 \\
\end{array}$ & $19 \%$ & None \\
\hline 26 & $\begin{array}{c}\text { China } \\
\text { (mainland) }\end{array}$ & VAT & - & $\begin{array}{l}\text { January } \\
1994\end{array}$ & $6 \%$ to $16 \%$ & $\begin{array}{c}\text { CNY5,000 to } \\
\text { CNY20,000 } \\
\text { (Monthly) }\end{array}$ \\
\hline 27 & Colombia $^{8}$ & VAT & $\begin{array}{c}\text { IVA - Impuesto sobre } \\
\text { las Ventas }\end{array}$ & $\begin{array}{c}\text { December } \\
1983\end{array}$ & $19 \%$ & None \\
\hline 28 & Cook Islands & VAT & $\begin{array}{l}\text { Similar to OECD } \\
\text { model }\end{array}$ & 1997 & $15 \%$ & $\$ 30,000$ \\
\hline 29 & Costa Rica $^{9}$ & VAT & -- & $\begin{array}{l}\text { December } \\
2018\end{array}$ & $13 \%$ & None \\
\hline 30 & Croatia & VAT & $\begin{array}{c}\text { PDV - Porez na } \\
\text { Dodanu Vrinjednost }\end{array}$ & $\begin{array}{c}\text { January } \\
1998\end{array}$ & $25 \%$ & $\begin{array}{l}\text { HRK300,000 } \\
\text { (Business }\end{array}$ \\
\hline
\end{tabular}

${ }^{7}$ Standard Rate is presently reduced to $50 \%$ due to COVID-19

${ }^{8}$ Has reduced VAT rate to 0\% for Hospitality, restaurants and cafes due to COVID-19 till 31 December 2020

${ }^{9}$ Has reduced VAT rate to $9 \%$ due to COVID-19 
Dr. Hariharan Narayanan

\begin{tabular}{|c|c|c|c|c|c|c|}
\hline & & & & & & $\begin{array}{c}\text { established in } \\
\text { country) }\end{array}$ \\
\hline 31 & Curacao & TOT & Turnover Tax & March 1999 & $6 \%$ & None \\
\hline 32 & Cyprus $^{10}$ & VAT & -- & July 1992 & $19 \%$ & EUR35,000 \\
\hline 33 & $\begin{array}{c}\text { Czech } \\
\text { Republic }^{11}\end{array}$ & VAT & -- & $\begin{array}{c}\text { January } \\
1993\end{array}$ & $21 \%$ & CZK01 million \\
\hline 34 & Denmark & VAT & -- & July 1967 & $25 \%$ & $\begin{array}{c}\text { DKK50,000 } \\
\text { (Business } \\
\text { established in the } \\
\text { country) }\end{array}$ \\
\hline 35 & $\begin{array}{c}\text { Dominican } \\
\text { Republic }\end{array}$ & Tax & $\begin{array}{l}\text { Tax on the Transfer } \\
\text { of Industrialized } \\
\text { Goods and Services } \\
\text { ITBIS - Local name }\end{array}$ & May 1992 & $18 \%$ & None \\
\hline 36 & Ecuador & VAT & $\begin{array}{l}\text { IVA - Impuesto al } \\
\text { Valor Agregado }\end{array}$ & $\begin{array}{c}\text { December } \\
1981\end{array}$ & $12 \%$ & None \\
\hline 37 & Egypt & VAT & -- & $\begin{array}{c}\text { September } \\
2016\end{array}$ & $14 \%$ & EGP500,000 \\
\hline 38 & El Salvador & VAT & $\begin{array}{l}\text { ITBMS - Impuesto a } \\
\text { la Transferences de } \\
\text { Bienes Muebles y a la } \\
\text { prestacion de } \\
\text { Servicios }\end{array}$ & July 1992 & $13 \%$ & USD5,714.29 \\
\hline 39 & Estonia & VAT & -- & $\begin{array}{c}\text { January } \\
1991\end{array}$ & $20 \%$ & EUR40,000 \\
\hline 40 & Ethiopia & VAT & -- & $\begin{array}{c}\text { January } \\
2003 \\
\end{array}$ & $15 \%$ & ETB01 million \\
\hline 41 & Fiji & VAT & -- & July 1992 & $9 \%$ & FJD 100,000 \\
\hline 42 & Finland & VAT & & June 1994 & $24 \%$ & EUR10,000 \\
\hline 43 & France & VAT & $\begin{array}{l}\text { TVA - Taxe sur la } \\
\text { Valeur Ajoutee }\end{array}$ & April 1954 & $20 \%$ & None \\
\hline 44 & Georgia & VAT & -- & $\begin{array}{c}\text { December } \\
1993\end{array}$ & $18 \%$ & $\begin{array}{l}\text { GEL100,000 } \\
\text { (Business } \\
\text { established in } \\
\text { country) }\end{array}$ \\
\hline 45 & Germany $^{12}$ & VAT & -- & $\begin{array}{c}\text { January } \\
1968\end{array}$ & $19 \%$ & None \\
\hline 46 & Ghana & VAT & -- & March 1998 & $12.5 \%$ & GHS200,000 \\
\hline 47 & Greece $^{13}$ & VAT & $\begin{array}{c}\text { FPA - Foros } \\
\text { Prostithemenis } \\
\text { Aksias }\end{array}$ & $\begin{array}{c}\text { January } \\
1987\end{array}$ & $24 \%$ & None \\
\hline 48 & Guam & VAT & Sales Tax Rate & 2014 & $2 \%$ & -- \\
\hline 49 & Guatemala & VAT & $\begin{array}{l}\text { IVA - Impuesto al } \\
\text { Valor Agregado }\end{array}$ & July 1992 & $12 \%$ & None \\
\hline
\end{tabular}

${ }^{10}$ Has reduced VAT rate to 5\% due to COVID-19 from 1 July 2020 to 10 January 2021, for hotel, accommodation, hospitality, restaurants, cafes and public transport

${ }^{11}$ Has reduced VATT rate to $10 \%$ from 1 July 2020 to 31 December 2020 due to COVID-19 for accommodation, sports and cultural activities

${ }^{12}$ Has reduced the Standard Rate to $16 \%$ from 1 July 2020 to 31 December 2020, due to COVID-19

${ }^{13}$ Has reduced the Standard Rate to 13\% for Public Transport, taxis, ferries from 1 June 2020 to 31 October 2020 due to COVID-19 
Value Added Tax - Vat A Centenarian [1920-2020] in This Covid-19

\begin{tabular}{|c|c|c|c|c|c|c|}
\hline 50 & Guinea & VAT & -- & & $15 \%$ & GNF 500 million \\
\hline 51 & $\begin{array}{c}\text { Gulf } \\
\text { Cooperation } \\
\text { Council } \\
\text { [Bahrain } \\
\text { Saudi Arabia } \\
\text { Kuwait } \\
\text { Oman } \\
\text { Qatar } \\
\text { UAE] }\end{array}$ & VAT & -- & $\begin{array}{c}\text { Saudi Arabia } \\
\text { and UAE - } \\
\text { January } \\
2018 \\
\text { Bahrain } \\
\text { January } \\
2019\end{array}$ & $5 \%$ & SAR375,000 \\
\hline 52 & Guyana & VAT & -- & $\begin{array}{c}\text { January } \\
2007\end{array}$ & $14 \%$ & G\$ 15 million \\
\hline 53 & Honduras & $\begin{array}{l}\text { VAT or } \\
\text { Sales Tax }\end{array}$ & -- & $\begin{array}{c}\text { January } \\
1964\end{array}$ & $15 \%$ & None \\
\hline 54 & Hungary & VAT & -- & $\begin{array}{c}\text { January } \\
1988\end{array}$ & $27 \%$ & $\begin{array}{l}\text { For all taxable } \\
\text { person }\end{array}$ \\
\hline 55 & Iceland & VAT & -- & $\begin{array}{c}\text { January } \\
1990\end{array}$ & $24 \%$ & ISK02 million \\
\hline 56 & India & $\begin{array}{l}\text { GST and } \\
\text { Customs } \\
\text { Duty }\end{array}$ & -- & $\begin{array}{c}\text { GST } \\
\text { July } 2017 \\
\text { Customs } \\
\text { Duty } \\
1962 \\
\end{array}$ & $\begin{array}{c}\text { GST } \\
\text { From 5\% to } \\
28 \%\end{array}$ & $\begin{array}{c}\text { INR01 million } \\
\text { (Special Category } \\
\text { States specified) } \\
02 \text { million (Other } \\
\text { States) }\end{array}$ \\
\hline 57 & Indonesia & VAT & $\begin{array}{c}\text { PPN - Pajak } \\
\text { Pertambahan Nilai }\end{array}$ & $\begin{array}{c}\text { January } \\
1984\end{array}$ & $10 \%$ & $\begin{array}{l}\text { IDR4.8 billion } \\
\text { (Small } \\
\text { enterprises) } \\
\end{array}$ \\
\hline 58 & Iran & VAT & -- & $\begin{array}{l}\text { September } \\
2008\end{array}$ & $9 \%$ & -- \\
\hline 59 & $\begin{array}{l}\text { Ireland }^{14} \\
\text { Republic of }\end{array}$ & VAT & -- & $\begin{array}{c}\text { November } \\
1972\end{array}$ & $23 \%$ & $\begin{array}{c}\text { EUR37,500 } \\
\text { (Supplying } \\
\text { Services) to } \\
\text { EUR75,000 } \\
\text { (Supplying } \\
\text { Goods) }\end{array}$ \\
\hline 60 & Isle of Man & VAT & -- & April 1973 & $20 \%$ & $\begin{array}{c}\text { GBP85,000 } \\
\text { (Established } \\
\text { Business) }\end{array}$ \\
\hline 61 & Israel & VAT & & July 1976 & $17 \%$ & ILS99,003 \\
\hline 62 & Italy & VAT & $\begin{array}{l}\text { IVA - Imposta sul } \\
\text { Valore Aggiunto }\end{array}$ & $\begin{array}{c}\text { January } \\
1973 \\
\end{array}$ & $22 \%$ & None \\
\hline 63 & Jamaica & GCT & $\begin{array}{c}\text { General Consumption } \\
\text { Tax } \\
\end{array}$ & 1991 & $15 \%$ & $\$ 10$ million \\
\hline 64 & Japan & CT & $\begin{array}{c}\text { CT - Consumption } \\
\text { Tax } \\
\end{array}$ & April 1989 & $10 \%$ & JPY10 million \\
\hline 65 & $\begin{array}{l}\text { Jersey, Channel } \\
\text { Islands }\end{array}$ & GST & -- & May 2008 & $5 \%$ & JEP300,000 \\
\hline 66 & Jordan & $\begin{array}{l}\text { GST } \\
\text { GT } \\
\text { ST }\end{array}$ & $\begin{array}{c}\text { General Sales Tax } \\
\text { General Tax } \\
\text { Special Tax }\end{array}$ & June 1994 & $\begin{array}{l}\text { For GT 16\% } \\
\text { For ST } \\
\text { Various rates }\end{array}$ & $\begin{array}{l}\text { JOD10,000 to } \\
\text { JOD75,000 } \\
\text { (Depends on } \\
\text { Business) }\end{array}$ \\
\hline
\end{tabular}

${ }^{14}$ Has reduced the Standard Rate to 21\% from 1 September 2020 to 28 February 2021, due to COVID-19 International Journal of Research -GRANTHAALAYAH 
Dr. Hariharan Narayanan

\begin{tabular}{|c|c|c|c|c|c|c|}
\hline 67 & Kazakhstan & VAT & $\begin{array}{c}\text { NDS - Nalog na } \\
\text { Dobavlennuyu } \\
\text { Stoimost } \\
\text { KKS - Kosylgan Kun } \\
\text { Salygy }\end{array}$ & $\begin{array}{c}\text { December } \\
1991\end{array}$ & $12 \%$ & $\begin{array}{c}\text { USD200,000 for } \\
2019\end{array}$ \\
\hline 68 & Kenya $^{15}$ & VAT & -- & $\begin{array}{c}\text { January } \\
1990\end{array}$ & $16 \%$ & KES05 million \\
\hline 69 & Korea & VAT & -- & July 1977 & $10 \%$ & None \\
\hline 70 & Kosovo & VAT & $\begin{array}{l}\text { TVSH - Tatimi mbi } \\
\text { Vleren e Shtuar }\end{array}$ & May 2001 & $18 \%$ & EUR30,000 \\
\hline 71 & Kyrgyzstan & VAT & -- & -- & $12 \%$ & KGS 08 million \\
\hline 72 & Latvia & VAT & -- & May 1995 & $21 \%$ & $\begin{array}{l}\text { EUR40,000 } \\
\text { (Business } \\
\text { established in } \\
\text { Latvia) }\end{array}$ \\
\hline 73 & Lebanon & VAT & -- & $\begin{array}{l}\text { February } \\
2002\end{array}$ & $11 \%$ & LBP100 million \\
\hline 74 & Liberia & GST & VAT & 2010 & $10 \%$ & LRD 03 million \\
\hline 75 & $\begin{array}{l}\text { Liechtenstein } \\
\text { Principality of }\end{array}$ & $\begin{array}{l}\text { Swiss } \\
\text { VAT }\end{array}$ & -- & July 2012 & $7.7 \%$ & $\begin{array}{l}\text { Swiss VAT Act } \\
\text { followed }\end{array}$ \\
\hline 76 & Lithuania $^{16}$ & VAT & -- & May 1994 & $21 \%$ & EUR45,000 \\
\hline 77 & Luxembourg & VAT & $\begin{array}{l}\text { TVA - Taxe sur la } \\
\text { Valeur Ajoutee }\end{array}$ & August 1969 & $17 \%$ & None \\
\hline 78 & $\begin{array}{c}\text { Macedonia, } \\
\text { Former } \\
\text { Yugoslav } \\
\text { Republic of }\end{array}$ & VAT & -- & April 2000 & $18 \%$ & MKD01 million \\
\hline 79 & Madagascar & VAT & -- & 1962 & $20 \%$ & MGA200 million \\
\hline 80 & Malawi & VAT & -- & July 2016 & $16.5 \%$ & K10 million \\
\hline 81 & Malaysia $^{17}$ & SST & $\begin{array}{l}\text { Sales tax and Service } \\
\text { tax }\end{array}$ & $\begin{array}{c}\text { September } \\
2018 \text { (For } \\
\text { Both) }\end{array}$ & $\begin{array}{c}\text { Sales Tax - } \\
10 \% \\
\text { Service Tax - } \\
6 \%\end{array}$ & $\begin{array}{c}\text { RM500,000 (For } \\
\text { Both) }\end{array}$ \\
\hline 82 & Maldives & GST & $\begin{array}{c}\text { TGST - Tourism } \\
\text { goods and service tax }\end{array}$ & $\begin{array}{c}\text { October } \\
2011\end{array}$ & $6 \%$ & MVR01 million \\
\hline 83 & Malta & VAT & -- & $\begin{array}{c}\text { January } \\
1999\end{array}$ & $18 \%$ & None \\
\hline 84 & Mauritius & VAT & -- & $\begin{array}{c}\text { September } \\
1998\end{array}$ & $15 \%$ & MUR06 million \\
\hline 85 & Mexico $^{18}$ & VAT & $\begin{array}{l}\text { IVA - Impuesto al } \\
\text { Valor Agregado }\end{array}$ & $\begin{array}{c}\text { January } \\
1980 \\
\end{array}$ & $16 \%$ & None \\
\hline 86 & Moldova $^{19}$ & VAT & $\begin{array}{c}\text { TVA - Taxa pe } \\
\text { Valoarea Adaugata }\end{array}$ & July 1998 & $20 \%$ & MDL1.2 million \\
\hline 87 & Monaco & VAT & -- & $\begin{array}{c}\text { January } \\
1993\end{array}$ & $20 \%$ & USD 38,910 \\
\hline
\end{tabular}

${ }^{15}$ Has reduced the Standard Rate to 14\% from 1 April 2020 due to COVID-19

${ }^{16}$ Has reduced the Standard Rate to 9\% till 31 December 2020 due to COVID-19

${ }^{17}$ Has reduced the Standard Rate for Service to 0\% from 30 March 2020 to 30 June 2021

${ }^{18}$ Has reduced the Standard Rate to $10 \%$ due to COVID-19

${ }^{19}$ Has reduced the Standard Rate to 15\% for Hospitality, restaurants and cafes from 1 May 2020 to 31 December 2020 due to COVID-19 
Value Added Tax - Vat A Centenarian [1920-2020] in This Covid-19

\begin{tabular}{|c|c|c|c|c|c|c|}
\hline 88 & Mongolia & VAT & -- & July 1998 & $10 \%$ & MNT50 million \\
\hline 89 & Montenegro & VAT & -- & $\begin{array}{c}2001 \\
\text { Revised in } \\
2017 \\
\end{array}$ & $21 \%$ & $\begin{array}{l}\text { Above } 18,000 \\
\text { Euros }\end{array}$ \\
\hline 90 & Morocco & VAT & $\begin{array}{l}\text { TVA - Taxe sur la } \\
\text { Valeur Ajoutee }\end{array}$ & $\begin{array}{c}\text { January } \\
1986 \\
\end{array}$ & $20 \%$ & None \\
\hline 91 & Myanmar & $\mathrm{CT}$ & Commercial Tax & March 1990 & $8 \%$ & MMK50 million \\
\hline 92 & Namibia & VAT & -- & $\begin{array}{l}\text { November } \\
2020\end{array}$ & $15 \%$ & NAD500,000 \\
\hline 93 & Nepal & VAT & -- & 1998 & $13 \%$ & NPR 05 million \\
\hline 94 & Netherlands & VAT & $\begin{array}{c}\text { BTW - Belasting over } \\
\text { de Togevoegde } \\
\text { Waarde }\end{array}$ & $\begin{array}{c}\text { January } \\
1969\end{array}$ & $21 \%$ & None \\
\hline 95 & New Zealand & GST & -- & $\begin{array}{c}\text { October } \\
1986\end{array}$ & $15 \%$ & NZD60,000 \\
\hline 96 & Nicaragua & VAT & -- & $\begin{array}{l}\text { December } \\
1984 \text { and } \\
\text { revised in } \\
\text { May } 2003 \\
\end{array}$ & $15 \%$ & None \\
\hline 97 & Nigeria & VAT & -- & $\begin{array}{c}\text { December } \\
1993\end{array}$ & $7.5 \%$ & None \\
\hline 98 & Norway & VAT & -- & $\begin{array}{c}\text { January } \\
1970\end{array}$ & $25 \%$ & NOK50,000 \\
\hline 99 & Pakistan & ST & Sales Tax & $\begin{array}{c}\text { November } \\
1990\end{array}$ & $\begin{array}{c}\text { Standard Rates } \\
\text { of } 17 \% \text {, } \\
16 \%, 15 \% \text { and } \\
13 \% \text { for } \\
\text { different } \\
\text { categories }\end{array}$ & PKR10 million \\
\hline 100 & Panama & VAT & -- & $\begin{array}{c}\text { December } \\
1976\end{array}$ & $7 \%$ & USD36,000 \\
\hline 101 & $\begin{array}{l}\text { Papua New } \\
\text { Guinea }\end{array}$ & GST & -- & $\begin{array}{l}\text { January } \\
2004\end{array}$ & $10 \%$ & PGK250,000 \\
\hline 102 & Paraguay & VAT & $\begin{array}{l}\text { IVA - Impuesto al } \\
\text { Valor Agregado }\end{array}$ & July 1992 & $10 \%$ & None \\
\hline 103 & Peru & VAT & -- & August 1991 & $18 \%$ & None \\
\hline 104 & Philippines & VAT & - & $\begin{array}{c}\text { January } \\
1988\end{array}$ & $12 \%$ & PHP03 million \\
\hline 105 & Poland & VAT & -- & July 1993 & $23 \%$ & PLN200,000 \\
\hline 106 & Portugal & VAT & -- & $\begin{array}{c}\text { January } \\
1986\end{array}$ & $\begin{array}{l}23 \%, 22 \% \text { and } \\
18 \% \text { for } \\
\text { different } \\
\text { regions }\end{array}$ & None \\
\hline 107 & Puerto Rico & SUT & Sales and Use Tax & $\begin{array}{l}\text { November } \\
2006\end{array}$ & $\begin{array}{c}10.5 \%+1 \%= \\
11.5 \% 20\end{array}$ & None \\
\hline 108 & Qatar & GCC & -- & $\begin{array}{c}\text { January } \\
2020\end{array}$ & $5 \%$ & $\begin{array}{c}\text { Gulf Cooperation } \\
\text { Council }\end{array}$ \\
\hline 109 & Romania & VAT & -- & July 1993 & $19 \%$ & EUR88,500 \\
\hline 110 & $\begin{array}{c}\text { Russian } \\
\text { Federation }\end{array}$ & VAT & -- & $\begin{array}{c}\text { December } \\
1991\end{array}$ & $20 \%$ & None \\
\hline
\end{tabular}


Dr. Hariharan Narayanan

\begin{tabular}{|c|c|c|c|c|c|c|}
\hline 111 & Rwanda & VAT & -- & $\begin{array}{c}\text { January } \\
2001\end{array}$ & $18 \%$ & RWF20 million \\
\hline 112 & Saint Lucia & VAT & -- & $\begin{array}{c}\text { October } \\
2012\end{array}$ & $12.5 \%$ & XCD400,000 \\
\hline 113 & Saudi Arabia ${ }^{21}$ & VAT & -- & $\begin{array}{c}\text { January } \\
2018\end{array}$ & $5 \%$ & SAR375,000 \\
\hline 114 & Serbia & VAT & -- & $\begin{array}{c}\text { January } \\
2005\end{array}$ & $20 \%$ & RSD08 million \\
\hline 115 & Seychelles & VAT & -- & July 2012 & $15 \%$ & SR05 million \\
\hline 116 & Singapore & GST & -- & April 1994 & $7 \%$ & SGD01 million \\
\hline 117 & Sint Maarten & RT & Revenue Tax & $\begin{array}{c}\text { January } \\
1997\end{array}$ & $5 \%$ & None \\
\hline 118 & Slovak Republic & VAT & -- & $\begin{array}{c}\text { January } \\
1993\end{array}$ & $20 \%$ & EUR50,000 \\
\hline 119 & Slovenia & VAT & -- & July 1999 & $22 \%$ & $\begin{array}{c}\text { EUR50,000 (For } \\
\text { business } \\
\text { established } \\
\text { Slovenia) }\end{array}$ \\
\hline 120 & South Africa & VAT & -- & $\begin{array}{c}\text { September } \\
1991\end{array}$ & $15 \%$ & ZAR01 Million \\
\hline 121 & South Korea & VAT & -- & 1977 & $10 \%$ & None \\
\hline 122 & South Sudan & VAT & -- & 1991 & $20 \%$ & None \\
\hline 123 & Spain & VAT & -- & $\begin{array}{c}\text { January } \\
1986\end{array}$ & $21 \%$ & None \\
\hline 124 & Sri Lanka & VAT & -- & August 2002 & $8 \%$ & LKR 50 million \\
\hline 125 & St. Lucia & VAT & -- & 2012 & $12.5 \%$ & $\$ 180,000$ \\
\hline 126 & Suriname & TOT & Turnover Tax & $\begin{array}{c}\text { December } \\
1997\end{array}$ & $\begin{array}{l}10 \% \text { for goods } \\
\text { and } 8 \% \text { for } \\
\text { services }\end{array}$ & None \\
\hline 127 & Sweden & VAT & -- & $\begin{array}{c}\text { January } \\
1969\end{array}$ & $25 \%$ & SEK30,000 \\
\hline 128 & Switzerland & VAT & $\begin{array}{c}\text { MWST - } \\
\text { Mehrwertsteuer } \\
\text { TVA - Taxe sur la } \\
\text { Valeur Ajoutee } \\
\text { IVA - Imposta sul } \\
\text { valore Aggiunto }\end{array}$ & $\begin{array}{c}\text { January } \\
1995\end{array}$ & $7.7 \%$ & CHF100,000 \\
\hline 129 & Taiwan & $\begin{array}{c}\text { VAT and } \\
\text { GBRT }\end{array}$ & $\begin{array}{c}\text { Business Tax } \\
\text { including VAT and } \\
\text { Gross Business } \\
\text { Receipts Tax }\end{array}$ & $\begin{array}{c}\text { June } 1931 \\
\text { and revised } \\
\text { on December } \\
2015 \\
\end{array}$ & $5 \%$ & None \\
\hline 130 & Tanzania & VAT & - & $\begin{array}{c}\text { July } 1998 \\
\text { amended on } \\
\text { July } 2015\end{array}$ & $18 \%$ & TZS100 million \\
\hline 131 & Thailand & VAT & -- & $\begin{array}{c}\text { January } \\
1992\end{array}$ & $7 \%$ & THB1.8 million \\
\hline 132 & Togo & VAT & $\begin{array}{c}\text { Taxe sur la Valeur } \\
\text { Ajoutee }\end{array}$ & -- & $18 \%$ & FCFA 50 million \\
\hline
\end{tabular}

${ }^{21}$ Has increased the Standard Rate from 5\% to 15\% from 1 July 2020 due to COVID-19 International Journal of Research -GRANTHAALAYAH 
Value Added Tax - Vat A Centenarian [1920-2020] in This Covid-19

\begin{tabular}{|c|c|c|c|c|c|c|}
\hline 133 & $\begin{array}{c}\text { Trinidad and } \\
\text { Tobago }\end{array}$ & VAT & -- & $\begin{array}{c}\text { January } \\
1990\end{array}$ & $12.5 \%$ & $\begin{array}{c}\text { TTD500,000 } \\
\text { (from January } \\
2016 \text { ) }\end{array}$ \\
\hline 134 & Tunisia & VAT & -- & June 1988 & $19 \%$ & TND100,000 \\
\hline 135 & Turkey $^{22}$ & VAT & $\begin{array}{c}\text { KDV - Katma Deger } \\
\text { Vergisi }\end{array}$ & $\begin{array}{c}\text { November } \\
1984\end{array}$ & $18 \%$ & None \\
\hline 136 & Uganda & VAT & & July 1996 & $18 \%$ & UGX150 million \\
\hline 137 & Ukraine & VAT & $\begin{array}{l}\text { PDV - Podatok na } \\
\text { Dodanu Vartist }\end{array}$ & $\begin{array}{c}\text { January } \\
1992\end{array}$ & $20 \%$ & UAH01 million \\
\hline 138 & $\begin{array}{l}\text { United Arab } \\
\text { Emirates }\end{array}$ & VAT & -- & $\begin{array}{c}\text { January } \\
2018\end{array}$ & $5 \%$ & AED375,000 \\
\hline 139 & $\begin{array}{c}\text { United } \\
\text { Kingdom }^{23}\end{array}$ & VAT & -- & April 1973 & $20 \%$ & GBP85,000 \\
\hline 140 & United States & SUT & Sales and Use Tax & $\begin{array}{c}1930 \text { and } \\
1936\end{array}$ & $\begin{array}{c}\text { Range from } \\
2.9 \% \text { to } 7.25 \%\end{array}$ & $\begin{array}{l}45 \text { to } 50 \text { US } \\
\text { states follow }\end{array}$ \\
\hline 141 & Uruguay & VAT & -- & $\begin{array}{c}\text { December } \\
1972\end{array}$ & $22 \%$ & None \\
\hline 142 & Uzbekistan & VAT & -- & -- & $20 \%$ & -- \\
\hline 143 & Vanuatu & VAT & -- & 1998 & $12.5 \%$ & VUV 04 million \\
\hline 144 & Venezuela & VAT & -- & $\begin{array}{c}\text { October } \\
1993\end{array}$ & $16 \%$ & None \\
\hline 145 & Vietnam & VAT & -- & $\begin{array}{c}\text { January } \\
1999\end{array}$ & $10 \%$ & None \\
\hline 146 & Zambia & VAT & -- & July 1995 & $16 \%$ & ZMW800,000 \\
\hline 147 & Zimbabwe & VAT & -- & $\begin{array}{c}\text { January } \\
2004\end{array}$ & $15 \%$ & USD60,000 \\
\hline
\end{tabular}

Source: Compiled by Author from various secondary sources.

\subsection{VAT - ADOPTION ACROSS GLOBAL COUNTRIES}

(Cnossen, 1998) VAT has become one of the pillars of the tax system in over one hundred countries since late 1960s. It has become an inviting tax system because of the revenue generation. This study clearly states that properly designed and administered VAT system can generate more revenue with lower operational and economic costs. It also clearly states that the drawback and danger is that VAT becomes over-used in the sensitivity that it supplies to the care of an oversized, inefficient public sector.

(Cnossen, 2010) Three VAT studies: This study was separated into three parts, first concentrating on VAT for lawyers, economists, and accountants with special reference to Netherlands, second being examination and evaluation of VAT coordination in the EU against the backdrop of analysis of VAT fraud and finally the third discussing that value changes in exempt immovable property should be brought into the VAT footing identical to other second-hand goods that are bartered by taxable dealers. This three segmented part has made contribution towards better understanding of the VAT. First being the role of VAT correlating the views of lawyers, economists and accountants followed by VAT coordination aspects and the analysis of exempt immovable property and finally giving an evidence that domestic shadow economy fraud and contrived insolvency fraud are much more important than carousel fraud, which receives so much importance. This study is based on the papers presented at conferences held in Vienna, Washington DC and Oxford by Cnossen.

(Symons et al., 2010) VAT is now most common form of consumption tax system used around the world. This study was carried considering VAT and equivalent sales tax systems implemented in 145 different countries. The main objective was to look into the differences in the time required for VAT compliance in different countries. Data

\footnotetext{
${ }^{22}$ Has reduced the Standard Rate from 18\% to 1\% due to COVID-19 from 1 April 2020 to 30 November 2020

${ }^{23}$ Has reduced the Standard Rate from $20 \%$ to $0 \%$ for E-books and online journals, from $20 \%$ to $5 \%$ for Hospitality and tourism due to COVID-19 till January 2021
} 
from World Bank Group and PricewaterhouseCoopers LLP (PwC) were used for the study. The summary states that VAT is predominant and used throughout the world, time taken to comply with VAT is less in developed countries than developing countries, time taken to comply with VAT is more in countries where indirect taxes are not administered by the same tax authority that handles the corporate income tax, less time to comply in countries where business adopts online filing and payment of VAT, compliance takes longer time if documentation is more at the time of submission and there is correlation between VAT compliance and the time delay in receiving a VAT refund.

(Gerard \& Naritomi, 2018) This study says VAT has become one of the most important instruments of revenue mobilization in the developing world. VAT exists in almost more than 160 countries and roughly eighty percent of countries in sub-Saharan Africa have adopted VAT. It says that the key strengths of VAT systems are their selfenforcing properties. It also states that the legal system needs to be adapted to sufficiently support the new levels of information flowing into tax authorities. The layout of new policies should thoroughly consider 'take-up' costs in the local situation for consumers, firms and tax administrations.

(Evans et al., 2018) This study depends on the survey carried in the 47 member countries of the Forum on Tax Administration (FTA) that administer VAT or GST regime. It is a comparative cross-country assessments of the VAT compliance burden and its main drivers. The major findings were: VAT is considered "fit for purpose" as a vigorous instrument efficient of measuring and evaluating the business VAT compliance burden across the 47 FTA countries. It states that roughly $30 \%$ of the countries (14) have indications of a low to very low VAT compliance burden. The driving significant factors influence is grouped in the order, Policy Framework, administrative Framework and finally with capabilities of the revenue services with less prominence towards driving factors. It also clearly states that the VAT compliance burden is influenced by the economic development of the country and GDP.

(Alavuotunki et al., 2019) It highlights the influence of the debut of VAT on inequality and government revenues using anew released macro data. It states that with the accessible country-level inequality data one cannot evaluate those tax incidence impacts that could emerge from differences in the savings rates across people with different income levels. It also states that there is no evidence that the VAT would lead to widening welfare disparities.

(Blei, 2020) The standard VAT generally applies to all goods and services, unless advised differently by the legislation. The Standard rate in 2019 ranged from $27 \%$ in Hungary to less than $10 \%$ in Japan, Switzerland and Canada. In 2019 the average standard VAT rate of the 22 OECD countries which are member of the EU was at $21.8 \%$ which is notably higher than the OECD average.

\subsection{VAT - SPECIAL MEASURES DURING COVID-19}

(Deloitte, 2020) This speaks about high-level indicator of Covid-19 related VAT and sales tax measures introduced globally to mitigate the likely economic and fiscal effects of Covid-19 on business and individuals in various countries. It clearly specifies the countries which implemented measures towards VAT and also the types of measures adopted by each country during this Pandemic. The countries which have not implemented any measures are British Virgin Islands, Curacao, Indonesia, Mexico, New Zealand, Nicaragua, Serbia and Trinidal and Tobago. All other countries have implemented various measures. There are three types of measure implemented by various countries.

- Delayed or Spread Payment of VAT / Sales tax due and Delayed filing of VAT / Sales tax returns

- Reduction in rate of VAT and Sales tax rates and

- Other Measures.

The main measure implemented by almost all the countries are "Delayed or Spread payment of VAT/Sales tax due and Delayed filing of VAT/Sales tax returns". Some countries like Colombia, Czech Republic, France, Germany, Malaysia, Malta, Norway, Paraguay, Poland, Portugal, Puerto Rico, Slovenia, South Korea, Spain, Sweden, United Kingdom, have reduced the rate of VAT and Sales tax rates apart from delayed payment and delayed filing of VAT / Sales tax. Those countries which has implemented measures have included a term "other measures" which is more descriptive and followed in other means apart from the implantation of other two measures as mentioned above.

(ICC Statement, 2020) COVID-19 pandemic is an unusual health and economic crisis, disturbing the lives and livelihoods of workers, as well as the continued operations of businesses universally. MSMEs - Micro, Small and Medium-sized Enterprises and their workers, as well as entrepreneurs and the self-employed, are among the seriously hit. ICC - International Chamber of Commerce has highlighted number of measures which the government 
should take to save SMEs. The measures are quantified in a nutshell as: flexibility in extending deadlines for tax filing/payment, waiving of tax payments - both corporate income tax and indirect tax (Chile, Cyprus, Peru), flexible payment agreements with no interest or penalties (Chile, Cyprus, Peru), temporary reduction in VAT rates payable by small businesses (Republic of South Korea), 0\% tax on essential products (Brazil), Cash-flow assistance for MSMEs (Australia, Cook Islands, Bosnia, Herzegovina, France, South Africa.

(Commission, 2020a) Exemptions and reliefs towards Import duties and VAT are given for all those goods needed to combat the effects of the COVID-19 as per the request made by Italy, France, Germany, Spain, Austria, Cyprus, Czechia, Estonia, Greece, Croatia, Lithuania, Netherlands, Poland, Portugal, Slovenia, Belgium, Bulgaria, Denmark, Finland, Hungary, Ireland, Luxembourg, Latvia, Romania, Slovakia, United Kingdom, Sweden and Malta.

(KPMG (China) Limited, 2020) Exemptions and reliefs carried are quoted here: $100 \%$ tax depreciation for newly purchased equipment for manufacturing of Covid-19 prevention and control materials, VAT Exemption for income from transportation of Covid-19 prevention and control materials and refund of carried forward excess input VAT for manufacturers of Covid-19 prevention and control materials. The policy overviews mentions that, expanded scope for duty-free imported of donated goods-imported goods donated for Covid-19 prevention exempt from import duties, VAT and consumption Tax, Tax paid on donated imported goods will be refunded and Donated goods from US not subject to additional duties and tax paid will be refunded.

(Bulletin, 2020) Covid-19 pandemic has elicited a very different response when compared to previous financial crises. Many governments are modifying VAT/GST systems as an accelerated response measure to reinforce with financial liquidity. The impact of COVID-19 crisis on VAT/GST systems are reduction in business, customer default, practical challenges of operating in lockdown and repurposing production line. Many governments are providing relief for many affected businesses by extending the time limit of submission, reducing the rate of tax and providing all other measures to give life to the businesses.

\section{CONCLUSION}

VAT concept has emerged in 1920s and has expanded immensely across globe till date. The analysis makes it evident that the VAT rates are minimum at $2 \%$ in the country "Guam" and maximum at $27 \%$ in the country "Hungary", followed by Croatia, Denmark, Norway and Sweden with 25\%, Finland, Iceland and Greece with 24\%, Ireland and Poland with 23\%, Italy, Slovenia and Uruguay with 22\%, Argentina, Czech Republic, Latvia, Lithuania, Montenegro, Netherlands and Spain with 21\%. Apart from VAT these are the countries which have minimum and maximum rate of tax in their own systems. Aruba has minimum of 3\% for RT - Revenue Tax and HT - Health Tax, Canada with 5\% GST and Brazil has the highest tax bracket of $0 \%$ to $300 \%$ in the name of IPI - Federal VAT. Majority of countries which do not come into the bracket of VAT have adopted GST and other few countries have their own method of tax system which is more or less similar and equivalent to other global standards. During this COVID-19 era many countries have adopted tax rate reductions for all categories of goods and services and some countries have adopted for selective categories of good and services. The study identifies Austria, Bulgaria, Colombia, Costa Rica, Cyprus, Czech Republic, Germany, Greece, Ireland, Kenya, Lithuania, Malaysia, Mexico, Moldova, Turkey and United Kingdom are the countries which has reduced the tax rates as a measure of COVID-19 in order to make the public benefitted, whereas the only country which has taken a different route is Saudi Arabia which has increased the tax rate during this pandemic situation.

\section{AUTHOR'S SENSATION}

The author feels that this decrease or increase in VAT rates from the Standard Rate during this COVID-19 era will be an eye opener for all countries as to how best it can fit and match with the economy and benefit the public. This is a "New Model" which is developed in this challenging pandemic era and henceforth all countries will adopt such measures in the post Covid era considering this as a "Base Model".

VAT becomes a Centenarian in this COVID-19.

The author wishes many more returns for VAT without COVID INFLUENCE 


\section{VAT - A CENTENARIAN 1920-2020}

\section{SOURCES OF FUNDING}

This research received no specific grant from any funding agency in the public, commercial, or not-for-profit sectors.

\section{CONFLICT OF INTEREST}

The author have declared that no competing interests exist.

\section{ACKNOWLEDGMENT}

None.

\section{REFERENCES}

[1] Alavuotunki, K., Haapanen, M., \& Pirttilä, J. (2019). The Effects of the Value-Added Tax on Revenue and Inequality. Journal of Development https://doi.org/10.1080/00220388.2017.1400015

[2] Blei, F. (2020). Update June 2020. OECD Better Policies for Better Lives, 18(3), 284-318. https://doi.org/10.1089/lrb.2020.29087.fb

[3] Bulletin, T. P. (2020). COVID-19: The impact on VAT / GST systems. Pwc.

[4] Cnossen, S. (1998). Global Trends and Issues in Value Added Taxation. International Tax and Public Finance, 5, 399-428. https://doi.org/10.1023/A:1008694529567

[5] Cnossen, S. (2010). Three VAT Studies. In CPB Netherlands Bureau for Economic Policy Analysis.

[6] Commission, E. (2020a). Relief from Import Duties and VAT Exemptions for goods needed to combat the effects of the COVID-19. 4.

[7] Commission, E. (2020b). VAT rates applied in the Member States of the European Union. January (1).

[8] Covid-19 Related VAT and Sales Tax Measures Global summary. (2020). Deloitte, July.

[9] Ebrill, L., Keen, M., \& Summers, V. (2001). The Modern VAT. In the Modern VAT. https://doi.org/10.5089/9781589060265.071

[10] Ernst \& Young. (2019). Worldwide VAT, GST and Sales Tax Guide. 1-1072. https://www.ey.com/en_gl/taxguides/worldwide-vat-gst-and-sales-tax-guide-2019

[11] Evans, C., Highfield, R., Tran-nam, B., Walpole, M., \& Sydney, U. (2018). Diagnosing the VAT Compliance Burden: A Cross-Country Assessment. October, 1-53.

[12] Gerard, F., \& Naritomi, J. (2018). Value Added Tax in developing countries: Lessons from recent research VAT systems incentivise accurate reporting on B2B transactions, but evasion and administration challenges still exist. International Growth Centre, May.

[13] Limited, K. A. (China). (2020). Managing China Tax issues in Covid-19 disruption period. KPMG, February.

[14] Statement, I. C. C. (n.d.). ICC STATEMENT TAX MEASURES TO SAVE OUR SMES IN RESPONSE TO COVID-19. $1-3$.

[15] Symons, S., Howlett, N., \& Alcantara, K. R. (2010). The impact of VAT compliance on business. The World Bank/ International Finance Corporation; Price Water House Coopers, 25543(09/10), 1-15. 


\section{WEB LINKS}

[1] https://www.avalara.com/vatlive/en/vat-rates/international-vat-and-gst-rates.html

[2] https://en.wikipedia.org/wiki/Consumption_tax

[3] https://www.elibrary.imf.org/search?q=vat\&searchBtn=

[4] https://www.vat.international/brazil/

[5] https://www.worldbank.org/en/search?q=vat

[6] https://www.pwc.com/gx/en/tax/pdf/impact-of-vat.pdf

[7] http://www.oecd.org/ctp/tax-policy/tax-database-update-note.pdf

[8] https://www2.deloitte.com/content/dam/Deloitte/be/Documents/tax/COVID-19_TaxSurvey.pdf

[9] https://taxfoundation.org/digital-tax-europe-2020/

\section{ABOUT THE AUTHOR}

Dr. N. HARIHARAN is the Founder and Director of SHRI ACADEMY, Pune, Maharashtra, India. He is into Academics for more than 25 years serving in various capacities at reputed institutes teaching Core Finance and Business subjects at Undergraduate, Postgraduate and Professional Course levels in India and Abroad. He has attended and presented papers in various national and international conferences. $\mathrm{He}$ is also a Visiting Faculty in different B-Schools. He is member, board of studies/examiner in various universities and institutions. He is also member of Editorial Board of various Management Journals published from USA and India. He specializes in the field of Taxation, Accountancy and Finance. He has authored 12 editions of Income Tax Law and Practice and Income Tax Problems and Solutions (Indian Tax System). He has co-authored 02 books on Cost Accounting. He has guided more than 1000 projects for postgraduate courses for Indian and International Universities. His qualification is approved and recognized by BQA - Botswana

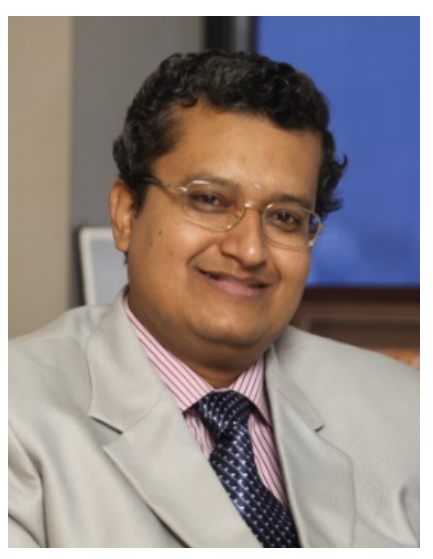
Qualifications Authority as equivalent to NCQF Level 10.

https://shriacademy.co.in/Home/Founder_Director 